\title{
Filter Banks, Wavelets, and Frames with Applications in Computer Vision and Image Processing (A Review) \\ invited
}

\author{
Ivar Austvoll \\ Stavanger University College, Department of Electrical Engineering and Computer \\ Science, Po.Box 8002, \\ N-4068 Stavanger, Norway \\ $\{$ Ivar.Austvoll\}@tn.his.no \\ http://ied.ux.his.no
}

\begin{abstract}
The purpose of this article is to present some of the fundamental principles of filter banks, wavelets and frames and their connections, with special emphasis on applications in computer vision and image processing. This is a vast field and we can only give a glimpse of it. We start with a short historical review and a rather broad discussion of filter banks, wavelets and frames. It is discussed how filter banks and wavelets are connected via multiresolution. Some of the most important structures and properties are presented but hardly no mathematical details are given. We focus especially on directional filter banks and wavelets, on analysis and extraction of directional features in images and image sequences. A system for motion estimation (estimation of optical flow) is presented.
\end{abstract}

\section{Introduction}

One of the primary tasks in computer vision is to extract features from an image or a sequence of images. The feature can be points, lines, edges, textures, velocity etc.. A given feature is characterized by position, direction, scale and other property parameters. The most common technique, used in early vision for the enhancement of such features, is linear filtering. This is also reflected in models used in biological visual systems, e.g. for human visual motion sensing see $[1,2]$. Another line of development is in digital processing of images, see $[3,4]$ and references therein. Objects in an image can have different sizes and therefore the scale of the linear filter is of great importance. Objects at different scales can arise from distinct physical processes. This leads to scale space filtering [5], multiresolution [7] and the use of many filters organized in filter banks or pyramids [6]. A single linear filter is described by its impulse response, a function in the continues domain or a sequence of filter coefficients in the discrete domain. The main issue is how to choose filter structure and impulse responses for the filters in the system to achieve a certain goal. A lot of work has been done but 
there are still no simple answer to this question. Here we will give an overview of some of the most common solutions suggested and applied to solve problems in computer vision and image processing.

The search for general image processing operators (linear filters) was a vivid research issue in the 1970's [9]. Filter banks used for sub-band decomposition of images has been known since the early 1980's [10]. ${ }^{1}$. The QMF (Quadrature Mirror Filter Bank) was first presented in [11]. These filter banks have been shown to be closely related to wavelets. Wavelets used for analysis of seismic signals was published by Morlet in 1983 [12]. The connection between filter banks, wavelets and multi-resolution signal analysis was recognized in the early 1990's [13,14]. Frames was first introduced in 1952 [15], but its use for signal representation was first connected with wavelets [16]. The interest for wavelets and frames has been increasing the last decade. The theory is well founded but there is still much work to be done on applications. Our interest is discrete signals in the form of images or image sequences. Subband compression of images is one of the most known applications of filter banks [17]. More recently the traditional filter bank has for some applications been replaced by filter banks implementing the wavelet transform or wavelet packets. We will not discuss this further in this paper, but affirm that in the JPEG 2000 still image compression standard the DCT (Discrete Cosine Transform) is replaced by the DWT (Discrete Wavelet Transform) implemented by a 2-band dyadic filter bank [19]. A comparison of different wavelet filters for image coding is found in [20]. Another important application of filter banks or wavelets is analysis of textures in images $[13,21$, $22]$. Filter banks and wavelets used for extraction of directional information like lines, edges and velocity will be discussed in section 3. In the next section we present some of the general principles and properties of filter banks, wavelets and frames. In section 4 an example of a system for estimation of motion is given. We end with a (preliminary) conclusion.

\section{Filter Banks, Multiresolution, Wavelets and Frames}

Filter banks and wavelets are connected. The connection is found via multiresolution $[13,14,18]$. We start with a short introduction to filter banks and use multiresolution as the link between filter banks and wavelets.

\section{$2.1 \quad$ Filter Banks}

We can define a digital filter bank [23], FB (= Filter Bank), as a collection of digital filters, with a common input or a common output. The purpose of the filter bank is usually to decompose the signal in sub-bands. Each filter, see Figure 1, outputs a portion of the signal given by the properties of the filter.

This system has been used extensively for compression. The filter bank used for decomposition is called the analysis bank and the filter bank used for

\footnotetext{
${ }^{1}$ Filter banks used for sub-band decomposition of acoustic signals was ten years earlier
} 


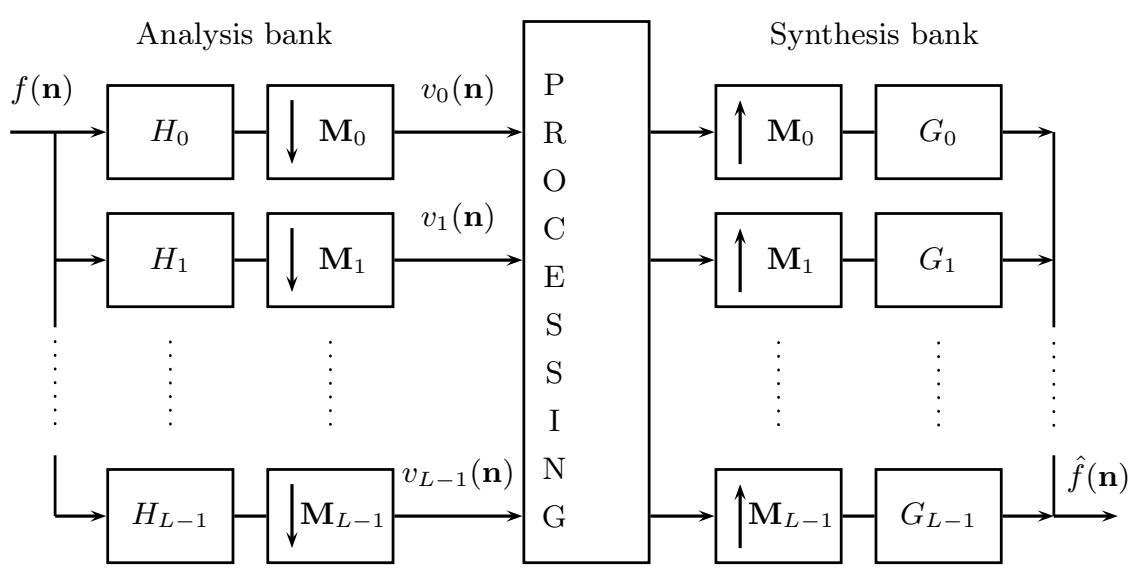

Fig. 1. L-channel multidimensional multirate filter bank. The input is a $D$-dimensional signal $f(\mathbf{n})$ where $\mathbf{n}=\left[n_{0} n_{1} \ldots n_{(D-1)}\right]^{T}, \quad n_{i} \in \mathbb{Z}, i \in \mathbb{Z}_{D}$ (The set of integers is $\mathbb{Z}$, and the integers in the range $\{0,1,2, \cdots, n-1\}$ is denoted $\left.\mathbb{Z}_{n}.\right) . H_{i}, G_{i}, i \in \mathbb{Z}_{D}$ are linear filters and the $\mathbf{M}_{i}, i \in \mathbb{Z}_{D}$ are decimator and expandor matrixes.

reconstruction is called the synthesis bank, see Figure 1. A necessary condition for compression is that it is possible to reconstruct the signal from the subbands. When the sub-band signals are coupled directly from the analysis bank to the synthesis bank, and the reconstructed signal is equal to the input signal ${ }^{2}, f(\mathbf{n})=\hat{f}(\mathbf{n})$, the filter bank is called a perfect reconstruction (PR) filter bank. The aliasing that occurs in the analysis FB is compensated for in the synthesis bank by proper design of the filters. Perfect reconstruction is also important in denoising (removal of noise) applications. In compression it is also important to keep the number of samples as low as possible. By using decimation (down sampling) of the sub-bands, it is possible to keep the total number of samples at the output of the analysis bank equal to the number of input samples. Then the filter bank is said to be maximally decimated. In this system we have at least two different sampling rates, it is therefore called a multidimensional multirate filter bank. The filter bank in Figure 1 is a general FB where the decimator (expandor) matrixes may be different for the different channels. For the most common rectangular sampling structure the $\mathbf{M}_{i}$ 's are diagonal ${ }^{3}$.

In other applications, e.g. texture analysis, feature extraction, motion estimation etc., only the analysis part is of interest. The design problem is therefore completely different from the compression case. We do not seek perfect recon-

${ }^{2}$ For images where $D=2$ we usually ignore the delay from input to output. For image sequences, $D=3$, the delay may be important for real time applications.

${ }^{3}$ For a quincunx grid we have $\mathbf{M}=\left[\begin{array}{cc}1 & 1 \\ -1 & 1\end{array}\right]$ and for a hexagonal structure $\mathbf{M}=\left[\begin{array}{cc}1 & 1 \\ 2 & -2\end{array}\right]$ 
struction but try to extract sub signals with certain features. The reduction of the number of samples is not necessarily important in such applications. In many such cases the decomposition will be redundant or over complete, i.e. we have more samples at the output than in the input signal.

By multiresolution we mean a decomposition in sub signals with different resolution. This is obtained by a non-uniform tiling of the frequency domain. For the $1 \mathrm{D}$ case this is usually done in octaves by reduction of the band-width by 2 at each step. This is achieved by a binary tree structured filter bank. For higher dimensions the possible number of ways to partition the frequency domain increases dramatically with the dimension, $D$. In the following discussion $D=2$.

In image processing, separable solutions based on tree structured filter banks are preferred in many applications. Separable filters reduce the computational complexity dramatically for higher dimensions. The result is a tiling of the frequency domain by rectangular-like sub-bands. This gives a simple implementation with low complexity. Theoretical analysis can be based on knowledge from $1 \mathrm{D}$ studies. A fully decimated 2D tree structured filter bank is shown in Figure 2. In computer vision a different approach is often chosen. This is motivated

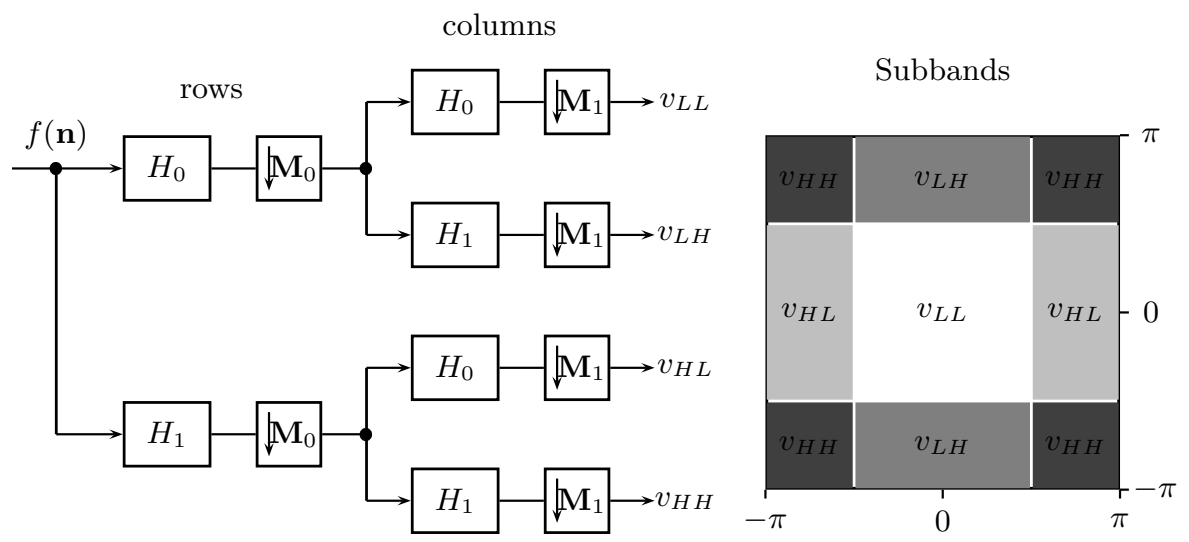

Fig. 2. Maximally decimated 2D tree-structured filter bank.

by the fact that real images, usually have no preferred direction, i.e. all directions should be treated equally. Circular sub-bands centered at the origin are therefore chosen. A simple structure for this is the Gaussian pyramid [6], Figure 3 4. The filter, $H_{G}$, is in this case a LP-filter ${ }^{5}$. Filter banks for the extraction of

4 The Laplacian pyramid has a similar structure but uses the high pass regions as well. The high pass signals are generated by the difference between the signal itself and a low pass signal found by using the filter $\left|H_{G}\right|^{2}$ 


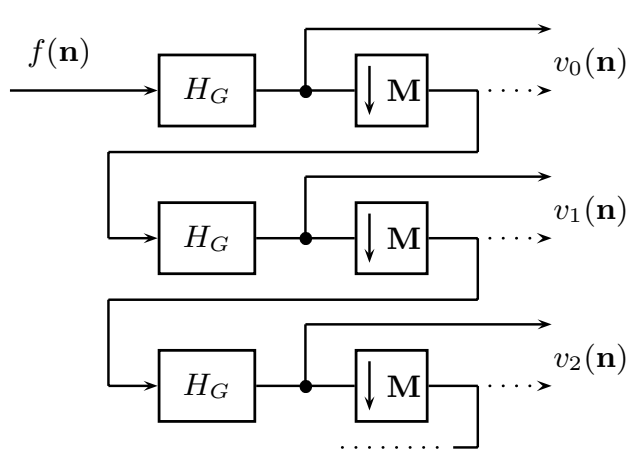

Subbands

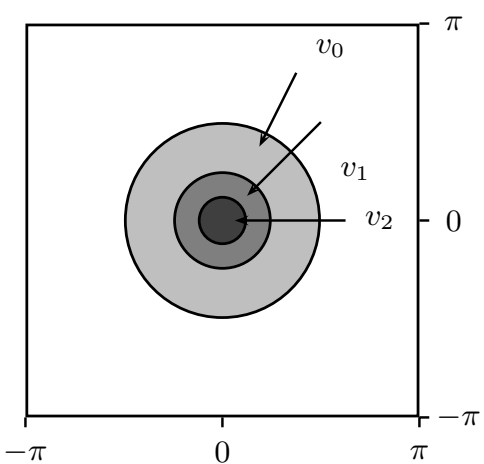

Fig. 3. Gaussian pyramid

directional information will be discussed in section 3 .

\section{$2.2 \quad$ Wavelets}

Only a brief discussion of wavelets will be given here. More details are found in the literature $[27,13,26,23]$. Most introductory work on wavelets are presented for one dimensional signals. As our interest is images we chose to start by writing an expression for the 2D Continuous Wavelet Transform (CWT):

$$
w_{f}(a, \mathbf{b})=\frac{1}{|a|} \int f(\mathbf{x}) \psi_{a, \mathbf{b}}\left(\frac{\mathbf{x}-\mathbf{b}}{a}\right) d \mathbf{x}
$$

where $\mathbf{x}=\left[\begin{array}{ll}x & y\end{array}\right]^{T}$ is the Cartesian coordinates, $a$ a scale parameter and $\mathbf{b}=$ $\left[\begin{array}{ll}b_{x} & b_{y}\end{array}\right]^{T}$ is a translation vector. The continuous function $\psi()$ is called the $2 \mathrm{D}$ mother wavelet. Equation (1) can be written as a convolution between the 2D signal $f(\mathbf{x})$ and a filter with impulse response given by the reflected and scaled mother wavelet. The scale parameter can be any real number. The result is therefore an infinite number of such convolutions. If we choose a finite number of scale parameters, $\left\{a_{0}, a_{1}, \cdots a_{L-1}\right\}$, we get $L$ convolutions, that can be put in the framework of an analysis filter bank. The result is a decomposition of the $2 \mathrm{D}$ signal in $L$ new signals, $w_{f}\left(a_{l}, \mathbf{b}\right), l \in \mathbb{Z}_{L}$ with properties given by the scaled "wavelet"-filters. In [28] the parameter space is extended with a rotation

${ }^{5}$ A traditional choice is a Gaussian filter. In our work on motion estimation we have used a filter with sharper transition $[8,25,24]$ with cut off approximately at $\pi / 2$. The decimation matrix for $2 \mathrm{D}$ is then $\mathbf{M}=\left[\begin{array}{ll}2 & 0 \\ 0 & 2\end{array}\right]$ 
parameter in addition to the scale parameter. Then the CWT is

$$
w_{f}(a, \theta, \mathbf{b})=\frac{1}{|a|} \int f(\mathbf{x}) \psi_{a, \theta, \mathbf{b}}\left(\frac{\mathbf{R}^{-1}(\mathbf{x}-\mathbf{b})}{a}\right) d \mathbf{x}
$$

where $\mathbf{R}$ is a $2 \mathrm{D}$ rotation matrix. The CWT in (1) transforms the $2 \mathrm{D}$ signal to $3 \mathrm{D}$, in $(2)$ to $4 \mathrm{D}$, it is therefore over-complete. The signal can be recovered by inverse filtering/deconvolution, or by the formulation of an Inverse Continuous Wavelet Transform (ICWT). This corresponds to the synthesis part in filter bank theory.

As for the filter bank we are first of all interested in the analysis of the signal. The important issue is the choice of a mother wavelet. Theory has been developed for the construction and design of wavelets with special properties. For image analysis the conditions formulated for many wavelets is in our view (also expressed in [28]) not necessary in many applications.

In digital implementations all continuous parameters must be sampled. Discretizing the parameters lead to the Wavelet Series (WS) ${ }^{6}$. This is usually done in the following way. Let $a=s^{k}$ and $\mathbf{b}=s^{k} \Delta x \mathbf{n}$, where $\mathbf{n}=\left[n_{x} n_{y}\right]^{T} \in \mathbb{Z}^{2}$ and $\Delta x$ is the size of a translation increment. The transform is still continuous, but results in a discrete parameter space (similar to the Fourier Series). For (2) and $\theta \in\left\{\theta_{0}, \theta_{1} \cdots \theta_{L-1}\right\}$ the result is a continuous filter bank ${ }^{7}$.

Sampling of the signal coordinates finally results in the Discrete Wavelet Transform (DWT) (analog to the DFT). A common case is to use $s=2, \Delta x=1$ and we replace the coordinates, $\mathbf{x}$, by the index vector $\mathbf{n}$ in equation (1). The result is

$$
w_{f}\left(2^{k}, \mathbf{n}\right)=2^{-k} \sum_{\mathbf{i}} f(\mathbf{i}) \psi_{\left(2^{k}, \mathbf{n}\right)}\left(2^{-k} \mathbf{i}-\mathbf{n}\right),
$$

where $\mathbf{i}$ is the summation index vector. Considered as a convolution this expression includes decimation with 2 in both coordinates and for each level. The signals, $w_{f}\left(2^{0}, \mathbf{n}\right), w_{f}\left(2^{1}, \mathbf{n}\right), \cdots$, represent "detail" or high frequency information in the signal. This wavelet transform (with binary scalings and dyadic translations of the mother wavelet), the DWT, can be implemented as a binary tree structured filter bank. The implementation can also be put in the same form as the Gaussian Pyramid with the addition of a "wavelet filter" connected to the signal at each level, after the decimator. This can also be seen as a recursion [26]. In [26] it is discussed, for what functions (wavelets), $\psi()$, this recursion is an exact implementation of (3) and how the DWT can be seen as an exact Wavelet Transform.

As mentioned above the analysis by wavelets can be implemented as a filter bank. The properties of the filter impulse responses must however satisfy certain conditions to represent wavelets. It is not quite obvious how important these properties are for many applications and this subject may seme somewhat obscure for many practitioners. In a recent article by Unser et. al [30] this

\footnotetext{
${ }^{6}$ In [23] this is called the Discrete Wavelet Transform

7 This is equivalent to the directional filter bank described in section $3[25,24]$
} 
has been discussed in an attempt to clarify the importance of some of these conditions. Their arguments are entirely based on B-splines [31] and fractional B-splines ${ }^{8}$. From a theoretical point of view the elementary building block of wavelets is the scaling function. In [30] it is shown that a valid scaling function is represented by the convolution between a B-spline and a distribution. The B-spline component leads to the primary mathematical wavelet properties order of approximation, reproduction of polynomials, vanishing moments and smoothness of the basis function. One of the consequences is that the analysis wavelet essentially behaves like a $\gamma$ th-order differentiator ${ }^{9}$

\section{$2.3 \quad$ Frames}

A nice introduction to discrete finite frames is found in [32]. Given a family of vectors $\left\{\mathbf{h}_{1}, \mathbf{h}_{2}, \ldots \mathbf{h}_{k} \ldots\right\}$ in a Hilbert space $H$. This family of vectors is called a frame if there exist real numbers $A, B>0$, such that for all $\mathbf{x} \in H$ :

$$
A\|\langle\mathbf{x}, \mathbf{x}\rangle\|^{2} \leq \sum_{k} \mid\left\langle\mathbf{x}, \mathbf{h}_{k}\right\rangle\left\|^{2} \leq B\right\|\langle\mathbf{x}, \mathbf{x}\rangle \|^{2} .
$$

$A, B$ are the frame bounds. The frame is called tight when $A=B$. If the family of vectors constitutes an orthonormal basis $A=B=1$. Frames are a generalization of bases. A signal can be represented by the bases vectors or the frame vectors by

$$
\mathbf{x}=\sum_{k} c_{k} \mathbf{h}_{k}
$$

Any collection of vectors $\left\{\mathbf{h}_{k}\right\}$ that span the actual space is a frame. It does not have to be orthogonal, and it is overcomplete. The expansion coefficients $\left\{c_{k}\right\}$ are therefore not unique. This give more freedom in the choice of vectors to represent the signal as compared to bases. The frame bounds are a measure of the redundancy of vectors for signal representation. Two important examples of discrete frames are the discrete Gabor ${ }^{10}$ expansion and the wavelet frames [32]. The connection between FBs, wavelets and frames are discussed in the next subsection.

\subsection{A Unified View on FBs, Wavelets and Frames}

Some of the connections between filter banks, wavelets and frames have been mentioned in the previous section. Here we will present this in the context of signal expansions [33] for $1 \mathrm{D}$ vectors. For a given signal vector $\mathbf{x}$ we write

$$
\mathbf{x}=\mathcal{H} \mathbf{w}=\sum_{k} w_{k} \mathbf{h}_{k} .
$$

\footnotetext{
${ }^{8}$ Splines are closely related to approximation of functions and interpolation of sampled data. Older then Shannons sampling theorem.

${ }^{9}$ Here differentiation is extended to fractional derivatives. In the frequency domain this can be described by $|\omega|^{\gamma}$ where $\gamma$ is a positive real number $\geq 1$.

10 Theory of communication. J.Inst.Elec.Eng., 93 (1946) 429-457
} 
This corresponds to the synthesis or reconstruction part of the filter bank approach. If the matrix $\mathcal{H}$ is invertible the expansion coefficients can be found by

$$
\mathbf{w}=\mathcal{H}^{-1} \mathbf{x}
$$

In many cases the inversion is not possible. This description can be used in the case of transforms, frames, wavelets and uniform FIR filter banks (or Lapped Orthogonal Transforms). The different cases can be considered as different structures of the nonzero elements in the matrix $\mathcal{H}$. This is illustrated in Figure 4 and Figure 5. The matrix $\mathcal{H}$ is band diagonal. Dots represent non zero elements in each sub matrix $\mathbf{H}$. In applications where the inversion of $\mathcal{H}$ is not

\section{Transform}

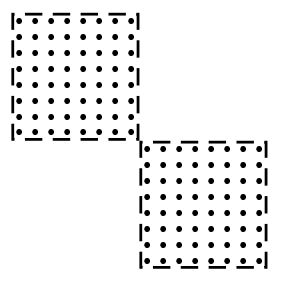

Wavelet

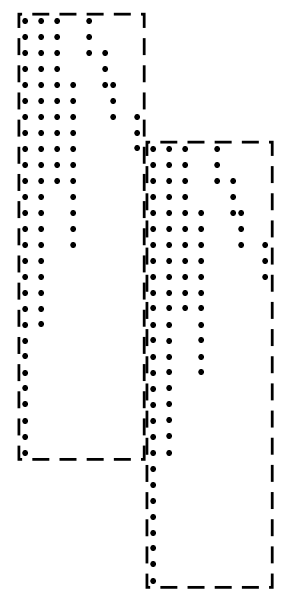

FB/LOT

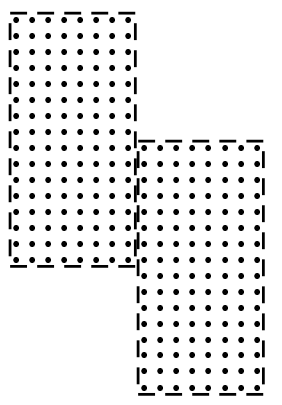

Fig. 4. The support structure for examples of a Transform, a Wavelet transform and a Filter Bank (Lapped Orthogonal Transform) represented by a band diagonal synthesis matrix $\mathcal{H}$. Each dashed box is a sub matrix $\mathbf{H}$ where dots represent non zero elements.

possible optimization methods can be used to find the expansion coefficients. This has been done for image compression [34] and sparse representations [35, 36]. In [35] a Frame Texture Classification Method is suggested. Small image frames are trained to represent different texture classes. Another application of frames is in denoising of images. In all these applications the goal is to find a sparse representation with as few nonzero elements in $\mathbf{w}$ as possible. 
Frame

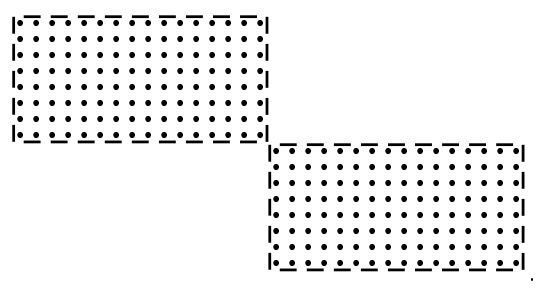

Overlapping Frame

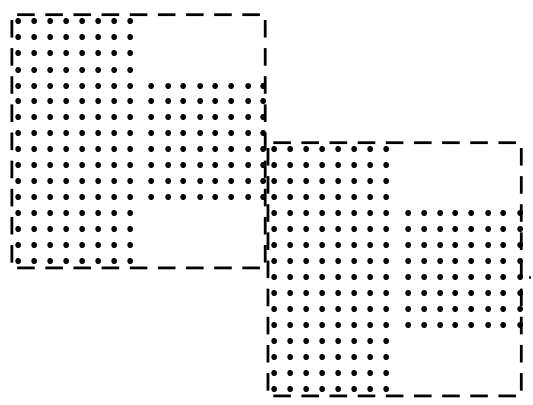

Fig. 5. The support structure for examples of a Frame and an Overlapping Frame represented by a band diagonal synthesis matrix $\mathcal{H}$. Each dashed box is a sub matrix $\mathbf{H}$ where dots represent non zero elements.

\section{Directional Filter Banks and Wavelets (Frames)}

In computer vision different types of directional analysis filter banks has been suggested. In $[37,39]$ a set of at least 3 quadrature filters is used to estimate the direction of an image structure. It was also suggested to use the same filters for texture analysis. A very popular set of filters is the localized spatial filters based on the Gabor functions. These where suggested by Daugman in the 1980's as a model for biological vision. In [41] it is suggested for texture analysis. A comparison between different texture classification methods based on filter banks and wavelets was presented in [22]. In this work it is shown that it is difficult to find a best approach in general. Some of the specialized filter banks give the best results in most of the cases. Both Gabor filters and common wavelet transforms are inferior to non dyadic decompositions and specialized filter banks.

An important feature in images and image sequences is edges and ridges. The common way of extending wavelets from $1 \mathrm{D}$ to $2 \mathrm{D}$ is to use the tensorproduct of one dimensional wavelets. This give good abilities in detecting point singularities i. e. across edges but will not be able to follow along the edge. For this purpose a better choice is the directional Gabor filters or two-dimensional directional wavelets [29] based on the Morlet wavelet or Cauchy wavelet. We have suggested a directional filter bank based on the discrete Prolate Spheriodal sequence (approximated by a Kaiser window) for decomposition of images in a system for estimation of optical flow [25, 24]. A more complicated continues wavelet transform, the Galilean wavelet, has been suggested [40] for tuning to four parameters, scale, position, spatial orientation and velocity. One of the problems with these approaches is a high degree of redundance and large computational complexity. 
A New development in the field of wavelets for computer vision and image processing was started with the work of Candès on ridgelets [42] and Candès and Donoho on curvelets [43] in 1998 and 1999 respectively. The observation was that the traditional wavelet transform is good at detecting singularities in one dimension, but do not capture the smooth structure along curves in two dimensions. The curvelet is based on a two step procedure, where the image first is decomposed in subbands and the ridgelet transform is used on each image block. The ridgelet transform is based on a rotation by the Radon transform and a one-dimensional wavelet decomposition. The success of the curvelet transform is mainly due to the principle of anisotropic scaling where width and length is related as width $=$ length ${ }^{2}$. The problem with this approach for digital images, in some applications, is a high degree of redundancy. A solution to this is suggested by Do in $[44,45]$. Here a two stage technique is used, first a Laplacian pyramid and then a directional filter bank. The result is a frame composed of contour segments called contourlets. Recently the finite ridgelet transform, FRIT, has been developed [46] (based on the finite Radon transform, FRAT). Application of the curvelet transform for image denoising is found in [47], and for speckle reduction of SAR images in [48].

\section{Estimation of Velocity and Optical flow}

The purpose of this section is to give an example of a system for estimation of optical flow based on three different filter banks combined. In Figure 6 the entire block-diagram for our motion estimation system is given. The system contains four different parts. The image sequence is first processed in the scale-space filters. The filtering is done in the spatial domain by a 2D LP (low pass) circular symmetric (non separable) filter with zero phase. This is equivalent to the system in Figure 3.

The output from each scale is down-sampled by 2 before the next filtering step. At each scale the LP-filtered sequence is sent to the directional filters. These filters are also 2D filters operating on each image in the sequence separately. Each filter is a complex filter with a directional BP (band pass) response. In Figure 7 the frequency responses for a set of directional filters are shown. The phase of the output from these filters is going to the third part of the system. Here space-time slices is taken for each direction represented by the directional filters. For those directions with highest energy a component velocity is found by using tensors computed from the output of a set of quadrature filters. As explained in [39] we need at least 3 filters to estimate direction in a 2D signal. More filters give more accurate results. We have found from experiments that four filters and more give good results. The improvement by going from 3 to 4 filters is appreciable, while for higher number of filters the improvement is small. Component velocities from lower levels are propagated to higher levels in a process using interpolation and compensation. The result is a set of component velocity fields that is used in the fourth part to compute a full velocity field or optical flow. More details and experimental results can be found in [25, 24]. 


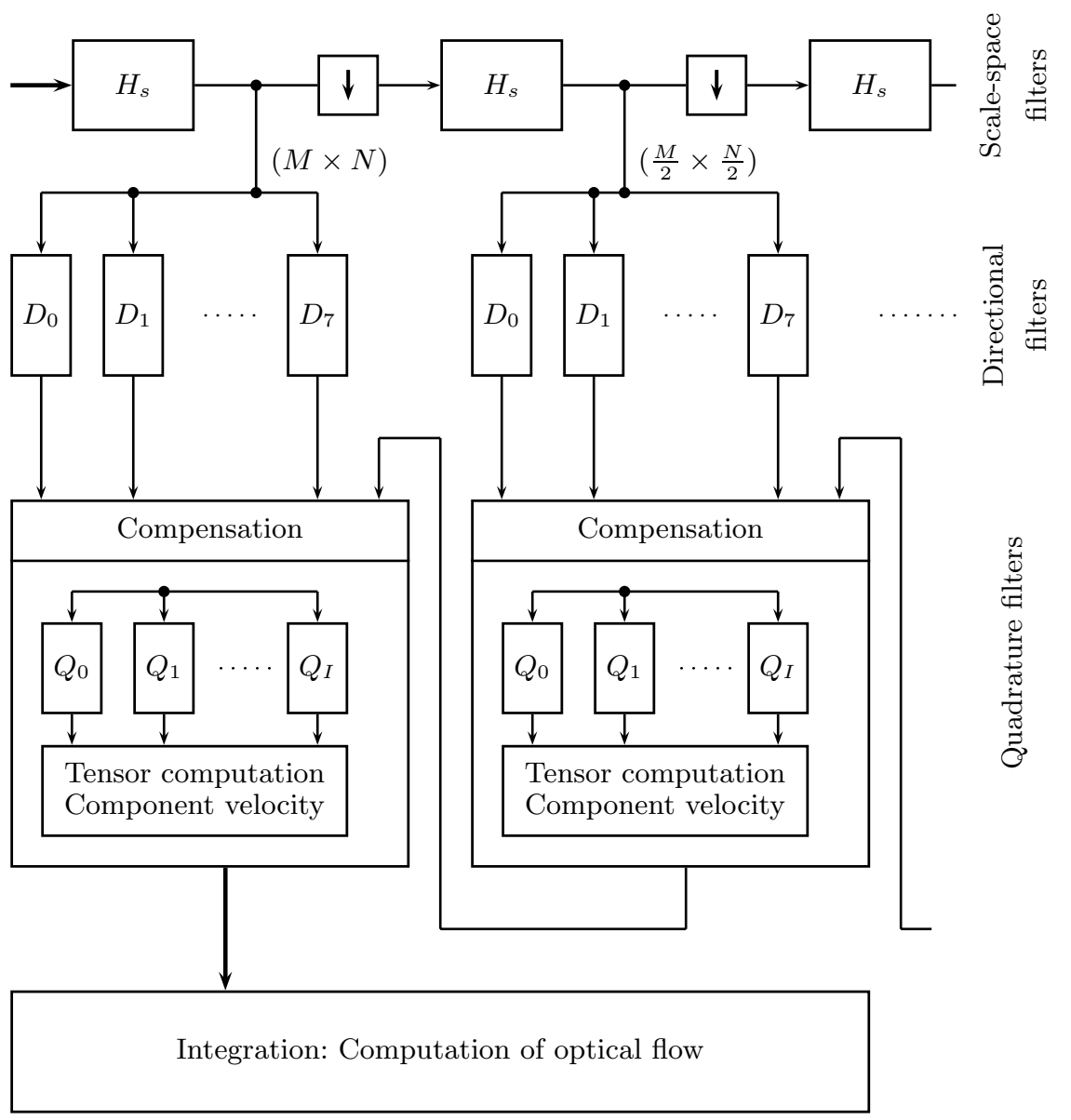

Fig. 6. Motion estimation system. 

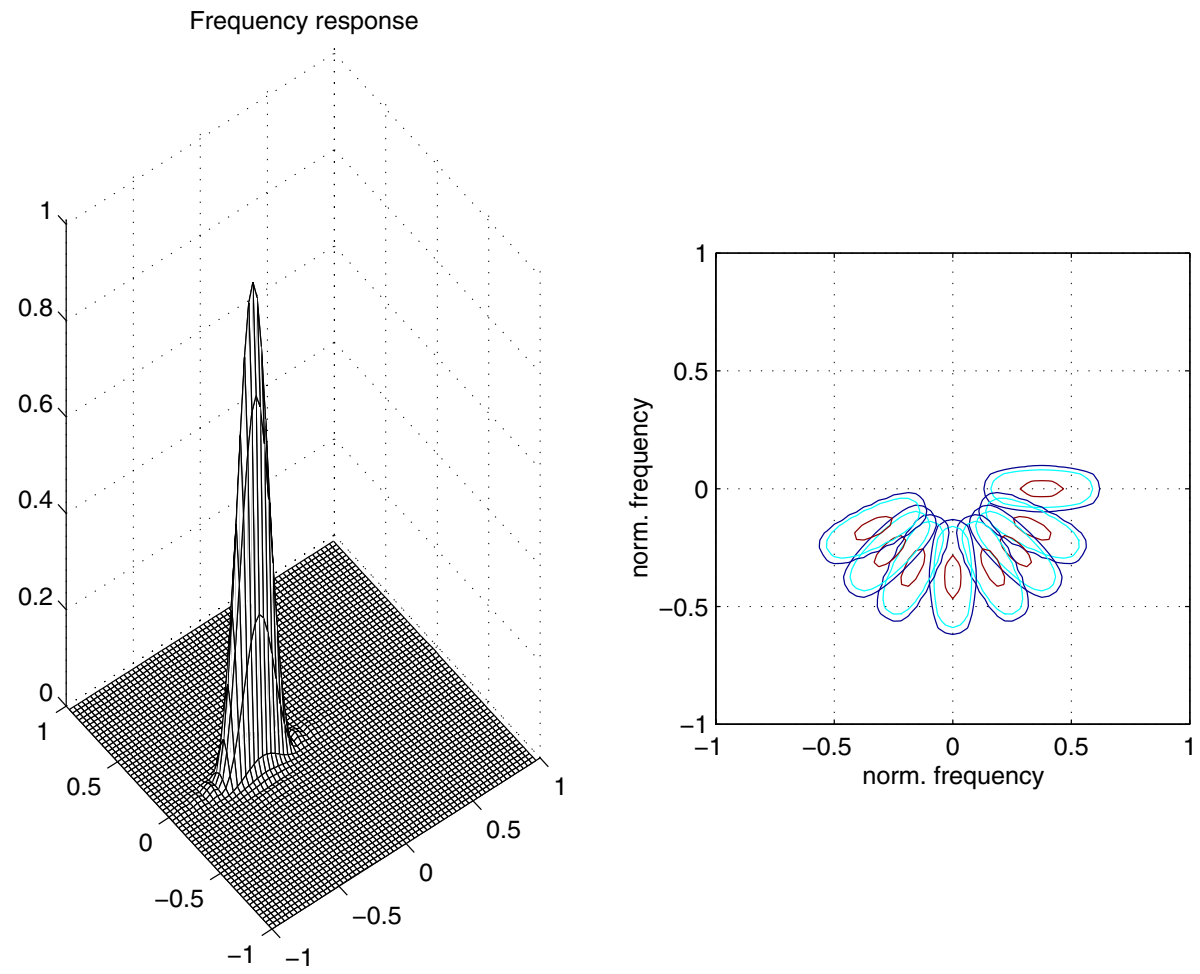

Fig. 7. Frequency response for directional filters based on the Kaiser window, where the bandwidth in the radial direction is twice the bandwidth in the transversal direction. The left plot shows the magnitude of the frequency response in direction number 4 . In the right part all filters are displayed together in a contour plot where the contours are at $-3,-20$ and $-30 \mathrm{~dB}$ respectively. We can see the slightly non uniform distribution of the filters due to our choice of directions. 


\section{Discussion and Conclusions}

The theoretical developments in the field of filter banks, wavelets and frames are formidable. Also a vast amount of applications have been published. The starting point has often been one dimensional signals and the results are extended to higher dimensions by separable solutions on a cartesian grid. As we have seen above, this is not favorable for images and image sequences. To get better results non separable solutions are usually needed. With the last developments in directional filter banks and directional wavelets a step forward is done in the effort to find good solutions for many computer vision applications. Computational complexity is an important issue for most applications. It is necessary to find good algorithms for discrete digital images. There are therefore several non trivial questions that has to be asked when we want to find good solutions to practical problems.

- What structure should be chosen for the decomposition?

- How many channels, how many subbands etc. ?

- What kind of filters or wavelets should be chosen ?

- How much redundancy is acceptable (or necessary to give accurate results) ?

For more complicated systems like the one described in section 4 there are too many degrees of freedom to do a full optimization with respect to accuracy of the result. We have found that a certain degree of redundancy is necessary to get accurate results in our system for motion estimation.

What is needed is more work, more experiments and comparative studies to find better solutions for important problems in computer vision, e.g. good descriptions for objects, disparity in stereo vision, estimation of velocity in image sequences etc..

Simple ad hoc methods are often dominant in the practical world. Why is it so difficult to find and introduce more modern and more accurate methods in practical applications?

\section{References}

[1] Watson, A. B., Ahumada, A. J. Jr.: Model of human visual-motion sensing, Journal of Optical Society of America 2 No. 2 (1985) 322-342

[2] Adelson, E. H. and Bergen, J. R.: Spatiotemporal energy models for the perception of motion, Journal of the Optical Society of America 2 No. 2 (1985) 284-299

[3] Jain, A. K.: Fundamentals of Digital Image Processing. Prentice-Hall, Englewood Cliffs (1989)

[4] Gonzales R. C., Woods R. E.: Digital Image Processing. Addison-Wesley Publishing Company (1993)

[5] Witkin, A.: Scale-space filtering. 8th International Joint Conference Artificial Intelligense, (1983) 1019-1022

[6] Burt, P. J.: The Pyramid as a Structure for Efficient Computation. Multi Resolution Image Processing and Analysis. (1984) Springer Verlag 6-35 
[7] Burt, P. J.: Multiresolution techniques for image representation, analysis, and 'smart' transmission. Visual Communications and Image Processing IV, SPIE 1199, (1989) 2-15

[8] Pauwels, E. J., Van Gool, L. J., Fiddelaers, P., Moons, T.: An Extended Class of Scale-Invariant and Recursive Scale Space Filters. IEEE Trans. Pattern Anal. and Machine Intell. 17 No. 7 (1995) 691-701

[9] Granlund, G. H.: In search of a general picture processing operator. Computer Graphics and Image Processing 8 No. 2 (1978) 155-178

[10] Woods J. W., O'Neill S. D.: Subband Coding of Images. IEEE Trans. Acoust., Speech, Signal Processing ASSP-34 No. 5 (1986) 1278-1288

[11] Esteban D., Galand C.: Application of quadarature mirror filters to split band voice coding schemes. Proc. Int. Conf. Acoust. Speech, Signal Proc. (1977) 191-195

[12] Morlet J.: Sampling theory and wave propagaation. NATO ASI Series 1, Issues in Acoustic signal/Image processing and recognition, Chen C. H.,ed., Springer Verlag, Berlin, 233-261

[13] Mallat, S.G.: A theory for multiresolution signal decomposition: the wavelet representation. IEEE Trans. Pattern Anal. and Machine Intell. 11 No. 7 (1989) 674-693

[14] Vetterli, M., Herley, C.: Wavelets and Filter Banks: Theory and Design. IEEE Trans. Signal Processing 40 No. 9 (1992) 2207-2232

[15] Duffin R.J., Shaeffer A.C.: A class of nonharmonic Fourier series. Trans.Amer.Math.Soc. 72 (1952) 341-366

[16] Daubechies, I.: Ten Lectures on Wavelets. Society for Industrial and Applied Mathematics, Philadelphia, Pennsylvania (1992)

[17] Ramstad, T.A., Aase, S.A., Hus $\varnothing y$, J.H.: Subband Compression of Images - Principles and Examples, Advances in Image Communication 6. ELSEVIER Science Publishers BV, North Holland (1995)

[18] Rioul, O.: A discrete-time multiresolution theory. IEEE Trans. Signal Processing 41 No. 8 (1993) 2591-2606

[19] Rabbani, M, Joshi, R.: An overview of the JPEG still image compression standard. Signal Processing: Image Communication 17 (2002) 3-48

[20] Winger L.L., Venetsanopoulos A.N.: Biorthogonal nearly coiflet wavelets for image compression. Signal Processing: Image Communication 16 (2001) 859-869

[21] Randen, T., Hus $\varnothing y$, J.H.: Optimal Texture Filtering. IEEE Int. Conf. on Image Proc. Proceedings, (1995), Washington, D.C., 374-377

[22] Randen, T., Husøy, J.H.: Filtering for Texture Classification: A Comparative Study. IEEE Trans. Pattern Anal. and Machine Intell. 21 No. 4 (1999) 291-310

[23] Vaidyanathan, P.P.: Multirate Systems and Filter Banks. Prentice Hall, Englewood Cliffs (1993)

[24] Austvoll, I.: Motion Estimation using Directional Filters. PhD thesis (1999) Stavanger University College (HIS)/The Norwegian University of Science and Technology (NTNU), PoBox 8002 Ullandhaug, N-4068 Stavanger, Norway.

[25] Austvoll, I.: Directional filters and a new structure for estimation of optical flow. IEEE Int. Conf. on Image Proc. Proceedings, (2000), Vancouver, Canada.

[26] Shensa, M.J.: The Discrete Wavelet Transform: Wedding the Á Trous and Mallat Algorithms. IEEE Trans. Signal Processing, 40 No. 10 (1992) 2464-2482

[27] Daubechies, I.: Orthonormal Bases of Compactly Supported Wavelets. Communications on Pure and Applied Mathematics, XLI (1988) 909-996

[28] Antoine, J.-P., Carrette, P.,Murenzi, R., Piette, B.: Image analysis with twodimensional continuous wavelet transform. Signal Processing, Elsevier, 31 (1993) $241-272$ 
[29] Antoine, J.-P., Murenzi R.: Two-dimensional directional wavelets and scale-angle representation. Signal Processing, Elsevier, 52 (1996) 259-281

[30] Unser M., Blu T.: Wavelet Theory Demystified. IEEE Trans. Signal Processing, 51 No. 2 (2003) 470-483

[31] Unser M.: Splines: A Perfect Fit for Signal and Image Processing. IEEE Signal Processing Magazine, 16 No. 6 (1999) 22-38

[32] Pei, S-C., Yeh, M-H.: An introduction to discrete finite frames. IEEE Signal Processing Magazine, 14 No. 6 (1997) 84-96

[33] Aase, S.A., Husøy, J.H., Skretting, K., Engan, K.: Optimized Signal Expansions for Sparse Representation. IEEE Trans. Signal Processing, 49 No. 5 (2001) 1087-1096

[34] Engan, K.: Frame Based Signal Representation and Compression. PhD thesis. (2000) Stavanger University College (HIS)/The Norwegian University of Science and Technology (NTNU), PoBox 8002 Ullandhaug, N-4068 Stavanger, Norway.

[35] Skretting, K.: Sparse Signal Representation using Overlapping Frames. PhD thesis. (2002) Stavanger University College (HIS)/The Norwegian University of Science and Technology (NTNU), PoBox 8002 Ullandhaug, N-4068 Stavanger, Norway.

[36] Skretting, K., Engan, K., Husøy, J.H., Aase, S.A.: Sparse representation of images using overlapping frames. Proc. 12th Scandianavian Conference on Image Analysis, SCIA 2001, Bergen, Norway (2001) 613-620

[37] Knutsson, H.: Filtering and Reconstruction in Image Processing. Diss. No. 88 (1982) Linköping University, S-581 83 Linköping, Sweden

[38] Knutsson, H., Granlund, G.H.: Texture Analysis Using Two-Dimensional Quadrature Filters. CAPAIDM Workshop, Pasadena, California Oct. (1983)

[39] Granlund, G.H., Knutsson, H.: Signal Processing for Computer Vision, Kluwer Academic Publishers (1995)

[40] Leduc, J.-P.: Spatio-temporal wavelet transforms for digital signal analysis. Signal Processing, 60, Elsevier (1997) 23-41

[41] Bovik, A. C., Clark, M., Geisler, W. S.: Multichannel Texture Analysis Using Localized Spatial Filters. IEEE Trans. Pattern Anal. and Machine Intell. 12 No. 1 (1990) 55-72

[42] Candés, E. J.: Ridgelets: Theory and applications. Ph.D. dissertation, (1998), Dept. Statistics, Stanford Univ., Stanford, CA, USA.

[43] Candés, E. J., Donoho, D. L.: Curvelets - a suprisingly effective nonadaptive representation for objects with edges. In Curve and Surface Fitting. Eds.: Cohen, A., Rabut, C., Schumaker, L. L., Saint-Malo, (1999) Vanderbilt University Press

[44] Do, M. N., Vetterli, M.: Pyramidal Directional Filter Banks and Curvelets. Proc. IEEE Int. Conf. on Image Proc. Proceedings, (2001), Thessaloniki, Greece.

[45] Do, M. N., Vetterli, M.: Contourlets: A Directional Multiresolution Image Representation. Proc. IEEE Int. Conf. on Image Proc. Proceedings, (2002), Rochester, New York

[46] Do, M. N., Vetterli, M.: The Finite Ridgelet Transform for Image Representation. IEEE Trans. Image Processing, 12 No. 1 (2003) 16-28

[47] Starck, J.-L., Candés, E. J., Donoho, D. L.: The Curvelet Transform for Image Denoising. IEEE Trans. Image Processing, 11 No. 6 (2002) 670-684

[48] Ulfarsson, M. O., Sveinsson, J. R., Benediktsson, J. A.: Speckle Reduction of SAR Images in The Curvelet Domain. Proc. IEEE Int. Conf. on Image Proc. Proceedings, (2002), Rochester, New York 